\title{
EFFECT OF POROSITY ON DEFORMATION, DAMAGE, AND FRACTURE OF CAST STEEL
}

\author{
Richard Hardin ${ }^{1}$, Christoph Beckermann ${ }^{1}$ \\ ${ }^{1}$ University of Iowa, Department of Mechanical and Industrial Engineering, 3131 SC, \\ Iowa City, IA 52242-1527
}

Keywords: Casting, Porosity, Tensile Properties, Damage, Fracture

\begin{abstract}
A combined experimental and computational study is performed to investigate the effect of internal shrinkage porosity on the mechanical behavior of cast steel under static loading. Steel plates containing various levels of porosity are cast in a sand mold, machined, and tensile tested until fracture. A significant loss of ductility is observed. Radiographic imaging is used to reconstruct the porosity field in the test specimens. The measured porosity field is then used in a finite-element stress analysis of the tensile tests. The local elastic properties are reduced according to the porosity fraction present and porous metal plasticity theory is used to model the damage due to porosity. Good agreement between measured and predicted stress-strain curves is obtained. The computational model proposed in this study allows for a detailed evaluation of the effect of porosity, including its size, shape and location, on the mechanical performance of a steel casting.
\end{abstract}

\section{Introduction}

Steel castings are under-utilized due to uncertainties in their performance and lack of expertise in casting design. Discontinuities in castings, like porosity, play an important role in casting underutilization. Porosity creates uncertainty in a design's robustness, since there are no methodologies for including its presence in the design. As a result designers employ overly large safety factors to entire parts to ensure reliability. Contributing to the issue, the processes of designing and producing castings are usually uncoupled, except for the specification of nondestructive evaluation (NDE) requirements. Unless design engineers have test data or experience for a part, they request NDE requirements without knowing how this relates to part performance. By predicting porosity accurately from casting simulation and realistically modeling its effects on the part performance, engineers can develop robust designs that are tolerant of the porosity and reliable. Engineering approaches have been applied here to simulate the effect of porosity on deformation, damage and fracture for a cast steel (WCB) in tensile tests.

ASTM A216 Grade WCB steel is a cast carbon steel having a combination of good ductility and strength, having a $248 \mathrm{MPa}$ (36 ksi) yield strength, $485 \mathrm{MPa}$ (70 ksi) tensile strength and 22\% elongation as minimum tensile requirements. Failure of such ductile metals occurs on the microscale by mechanisms of void nucleation, growth and coalescence. Voids can preexist as microporosity and nucleate from imperfections like second phase particles. After nucleation, voids grow from increasing hydrostatic stress and local plastic straining. As voids nucleate and grow, the void volume fraction, or damage, increases. The voids begin to interact, and the void volume fraction at which interaction between voids begins is the critical void volume fraction $f_{\mathrm{c}}$. As plastic strain continues to increase, local necking and coalescence occurs in the material 
between voids until a connected chain of voids forms and failure occurs. The void fraction at which fracture occurs is the failure void volume fraction $f_{\mathrm{F}}$.

The effects of porosity on the structural performance of steel castings on the macroscopic scale are not as well understood. In terms of stiffness, strength, fatigue and component performance in the presence of porosity [2,3], it is found that microporosity does not result in a measurable loss of stiffness, or large stress concentrations, or stress redistribution, but it greatly affects fatigue resistance [4-5]. The presence of microporosity reduces ductility of metals because microvoids pre-exist before the metal is stressed and the nucleation stage is bypassed. Macroporosity in metals causes gross section loss, and locally reduces their effective stiffness [6-8]. Macroporosity is not uniformly distributed throughout the entire cast part, and the casting material properties are not homogenous. Stress redistribution occurs in parts due to macropores, and stress concentrations occur near them that cause micro-cracks and failure. The mechanical behavior of porous materials can be categorized into three groups based on porosity amount [8]: less than $10 \%, 10 \%$ to $70 \%$, and materials greater than $70 \%$. This division is promoted because the materials at the extremes behave quite differently. The high porosity group is comprised of foams and cellular structures. The elastic-plastic behavior of the porous materials in the $10 \%$ to $70 \%$ porosity range has a non-linear dependence on the porosity amount [2,6-8]. Materials in the lowest range depend linearly on porosity, assuming that voids do not interact [9] and by considering isolated pores [10], or a uniform distribution of pores [11]. Applying the ductile failure micromechanical mechanisms described previously, one micromechanics-based model is the porous metal plasticity model. The finite element analysis (FEA) software ABAQUS has this model [10-13]. In the model, the volume fraction of porosity is a primary state variable, and the inelastic flow is modeled as voids grow and coalesce until failure occurs. Porous metal plasticity model is used here to predict the elastic-plastic behavior of cast steel with porosity.

Here castings with porosity were produced, made into specimens and radiographed. The porosity was quantitatively determined from the radiographs. The castings underwent tensile testing. Using porosity data from radiographs, finite-element models of the test specimens with porosity were created and the tensile testing simulated using elastic-plastic material models. Results of the simulated tensile behavior were compared with the measured tensile behavior.

\section{Experimental Procedures}

The effect of porosity on the elastic properties of steel was determined by testing cylindrical test specimens having $5 \mathrm{~mm}$ diameter gage section, and simulating the measurements. For the elastic property work castings were designed to position porosity in the specimen gage section [2]. Elastic properties were measured from stable cycle hysteresis loops during fatigue testing. For the elastic-plastic tensile property studies, WCB steel specimens were produced from $2.5 \mathrm{~mm}$ thick x $12.7 \mathrm{~cm}$ wide vertically cast plates of two lengths $(38.1 \mathrm{~cm}$ and $45.7 \mathrm{~cm})$. Five plates were produced at each length. The castings were normalized and tempered, and machined into $1.91 \mathrm{~cm}(0.75$ ”) thick tensile test coupons with a gage section width of $8.64 \mathrm{~cm}$ (3.4”). Other dimensions were determined by the ASTM E8 tensile test standard. Radiographs of the cylindrical/elastic property specimens were taken from orthogonal directions to reconstruct their porosity distributions in simulations [2]. For the flat plate tensile plates, radiographs as shown in Figure 1(a) were taken in which stepped gage blocks were placed. A calibration curve for radiographic gray level versus plate thickness was determined. Filtering and background corrections were performed and only indications identified as seen Figure 1(b) were analyzed for porosity. The thickness of the steel shown in Figure 1(c) was determined from the gray level 


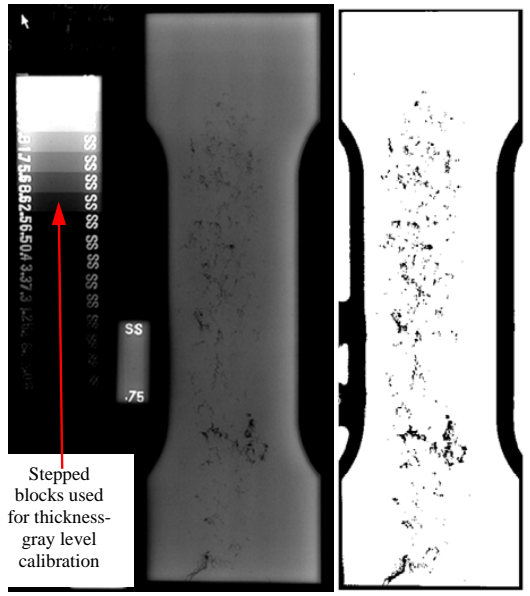

(a)

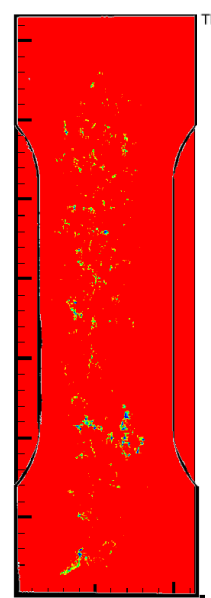

(c)

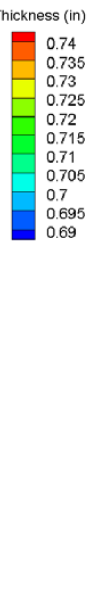

(b)

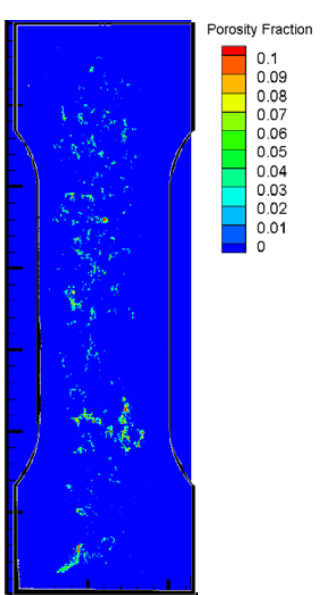

(d)

Figure 1 (a) Original radiograph image, (b) image of indications detected by analysis, (c) thickness measured from analysis and (d) section porosity measured in a test plate.

calibration curve, and division by the nominal (or "sound") machined plate thickness gave the porosity fraction through the plate (Figure 1(d)). Tensile testing was performed at the University of Iowa for the sound material, and for material with porosity at SSAB North American Division, Muscatine, IA. From the fracture surfaces after tensile testing, the porosity was found to lie about the mid-thickness of the plates. A finite element mesh for the tensile test plates was developed with mesh refinement at the plate mid-thickness shown in Figure 2(a). Mesh dimensions at the mid-thickness were defined so that two elements on each side about the midthickness of the plate were in a region $2.2 \mathrm{~mm}$ wide. This is a good representative value for the thickness of the porosity layer. The measured porosity (Figure 1(d)) is assumed to lie in this centerline region as seen in Figure 2(b). The porosity is scaled up accordingly and, in transferring measurements from the radiograph to the FEA nodes, the average porosity about a node position in the radiograph is determined, conserving porosity.

\section{Modeling Procedures Used}

The ABAQUS elastic material model is used below the yield stress with properties depending on porosity locally as a function of porosity fraction $f$ at the FEA nodes [2]. The porosity fraction is $f=V_{\text {pore }} / V_{0}$ where $V_{\text {pore }}$ is the volume of porosity in the sound metal matrix and $V_{0}$ is the total volume. The relationship between the elastic modulus and porosity fraction used node-by-node in the FEA analysis is $E(f)=E_{0}(1-f / 0.5)^{2.5}$ where $E_{0}=198 \mathrm{GPa}$ for WCB steel. The Poisson ratio $v$ was dependent on $f$ using $v(f)=v_{S}+\left(f / f_{\infty}\right)\left(v_{\infty}-v_{S}\right)$ with $v_{\infty}=0.14, f_{\infty}=0.472$ and the Possion ratio for the sound metal was taken as $v_{S}=0.3$.

The porous metal plasticity material model in ABAQUS is used to simulate ductile plasticity and failure. Due to space limitations, only a brief description can be given here. A complete 


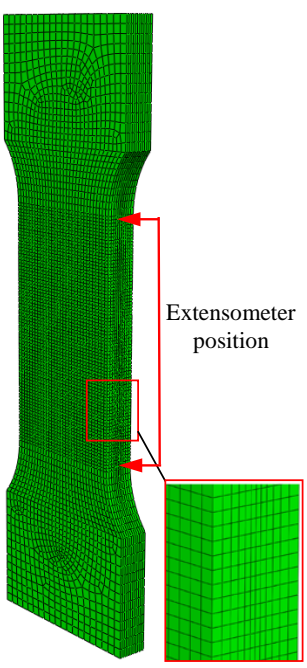

(a)

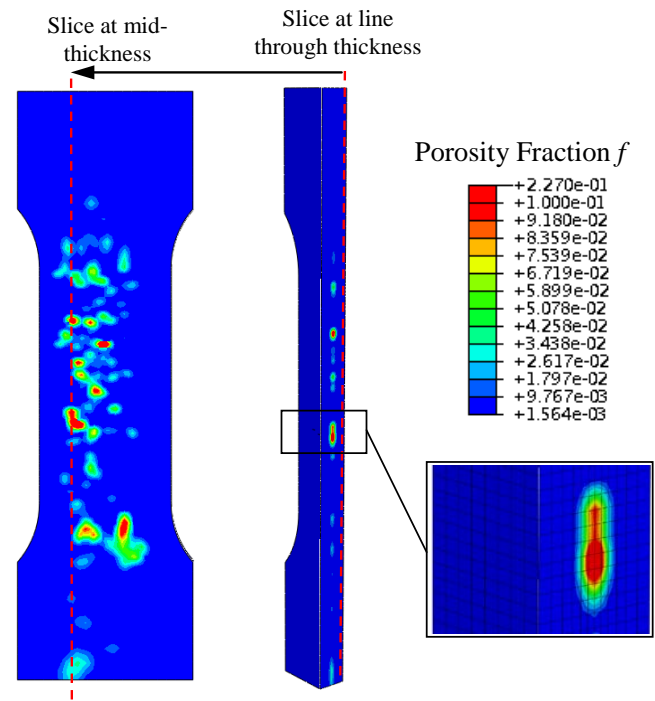

(b)

Figure 2 (a) Finite element mesh used in simulations of tensile test plates (b) example mapping the porosity faction to the mid-thickness plane of the FEA mesh.

presentation of the model is found in Gurson et al. [10-12], the software manual [13] and the numerical integration procedure used was developed by Aravas [14]. The model requires the user to define the hardening behavior of the metal matrix using true stress-strain data, which was determined from tensile tests for sound WCB steel without porosity. The yield function used in the model is given by

$$
\phi=\left(\frac{q}{\sigma_{y}}\right)^{2}+2 q_{1} f \cosh \left(-\frac{3}{2} \frac{q_{2} p}{\sigma_{y}}\right)-\left(1+q_{3} f^{2}\right)=0
$$

where $f$ is the porosity fraction, $q$ is the effective Von Mises Stress, $p$ is the hydrostatic stress, $\sigma_{y}$ is the yield stress of the fully sound material as a function of plastic strain, and $q_{1}, q_{2}$ and $q_{3}$ are material parameters. Note from Eq. (1) that when $f=0$ (for porosity free material), the yield condition becomes $q=\sigma_{y}$, or the Von Mises yield condition. The hydrostatic stress (or pressure) $p$ and the Von Mises Stress $q$ are the two stress invariants $p=-(1 / 3) \boldsymbol{\sigma}: \mathbf{I}$ and $q=\sqrt{(3 / 2) \mathbf{S}: \mathbf{S}}$ where $\boldsymbol{\sigma}$ is the Cauchy stress tensor and $\mathbf{S}$ is the deviatoric stress tensor $\mathbf{S}=p \mathbf{I}+$ $\boldsymbol{\sigma}$. The material parameters $q_{1}, q_{2}$ and $q_{3}$ in Eq. (1) are included to model the interactions between voids [11]. Values used here are $q_{1}=1.5, q_{2}=1.0$ and $q_{3}=2.25$, where $q_{3}=q_{1}^{2}$, as recommended for ductile metals [13]. The flow rule for the plastic strain rate $\dot{\varepsilon}^{p l}$

$$
\dot{\varepsilon}^{p l}=\dot{\lambda} \frac{\partial \phi}{\partial \boldsymbol{\sigma}}=\dot{\lambda}\left(-\frac{1}{3} \frac{\partial \phi}{\partial p} \mathbf{I}+\frac{3}{2 q} \frac{\partial \phi}{\partial q} \mathbf{S}\right)
$$

where $\dot{\lambda}$ is a non-negative scalar constant of proportionality, a measure of the plastic flow rate. The plastic strain in Eq. (2) causes damage, or void growth and nucleation, as it increases. The 
equation describing the growth rate of voids by growth and nucleation is $\dot{f}=(1-f) \dot{\varepsilon}_{k k}^{p l}+A \dot{\varepsilon}_{m}^{p l}$ where the first term on the right hand side denotes growth of existing voids from current void fraction $f$ and $\dot{\varepsilon}_{k k}^{p l}$, the total plastic strain rate (trace of the strain rate tensor), and the second term denotes the growth rate due to nucleation. In the nucleation term the equivalent plastic strain rate $\dot{\varepsilon}_{m}^{p l}$ is multiplied by a scaling coefficient $A=\frac{f_{N}}{s_{N} \sqrt{2 \pi}} \exp \left[-\frac{1}{2}\left(\frac{\varepsilon_{m}^{p l}-\varepsilon_{N}}{s_{N}}\right)^{2}\right]$ and is based on the assumption that the nucleation function $\left(\mathrm{A} / f_{N}\right)$ follows a normal distribution depending on the plastic strain range about a mean value $\varepsilon_{N}$, a standard deviation $s_{N}$ and a volume fraction of nucleated voids $f_{N}$. Values recommended for metals are used here [13]: $\varepsilon_{N}=0.3, s_{N}=0.1$ and $f_{N}=0.04$. A coalescence model and failure criteria model was used here [12,13], in which the void fraction $f$ in Eq. (1) is replaced by an effective void volume fraction due to coalescence $f^{*}$. In the equation, $f^{*}$ takes on the actual void volume fraction $f$ when it is less than the critical value $f_{c}$, where coalescence begins. When $f^{*}>f_{c}$, the effective void fraction increases more rapidly than $f$ due to the coalescence with $f^{*}=f_{c}+\frac{\bar{f}_{F}-f_{c}}{f_{F}-f_{c}}\left(f-f_{c}\right)$. In ABAQUS $\quad \bar{f}_{F} \quad$ is calculated from the model material parameters $\bar{f}_{F}=\left(q_{1}+\sqrt{q_{1}^{2}-q_{3}}\right) / q_{3}$. The material has no load carrying capacity when $f \geq f_{F}$, where $f_{F}$ is the void fraction at failure. In the present work $f_{c}=0.05$ and $f_{F}=0.15$. The purpose of the model description here is to give the most important model features and the parameters used. Details on the solution scheme used in ABAQUS is given in [13,14].

\section{Results}

Simulation results using the porosity dependent elastic properties are given in Figure 3. In Figure 3(a) the non-uniform stress-strain distribution for a test specimen is shown. Because of the nonuniformity, the predicted strain was averaged about the circumference of the gage section at the extensometer location, and its 95\% confidence interval was determined. Simulations for the 28 specimens are compared with measurements in Figure 3(b) showing good agreement for predicted versus measured strain. Note that where there is disagreement, there is also large uncertainty due to the non-uniformity in the measured strain.

Elastic-plastic tensile curves of the plate specimens with porosity were simulated using the porosity fields mapped to the FEA nodes at the plates' mid-thickness. The initial porosity fraction for the sound WCB steel was set to $0.2 \%$. This and the porous metal plasticity model parameters $\left(q_{1}=1.5, q_{2}=1.0, q_{3}=2.25, \varepsilon_{N}=0.3, s_{N}=0.1, f_{N}=0.04, f_{c}=0.05\right.$ and $\left.f_{F}=0.15\right)$ were determined by trial and error simulations, achieving good agreement between the measured and predicted sound tensile curves shown in Figure 4. The sound material's maximum elongation at failure, ultimate tensile (UT) stress, and yield stress are predicted within $2 \%$. Plates with porosity are simulated using the model parameters, and measured nodal porosity values or $0.2 \%$ porosity if $f=0$ at a node. In Figure 4, measured and predicted tensile curves for plate-ID E4 with porosity are shown. The reduction in ductility due to the porosity is well predicted. The yield stress for E4 is predicted to follow more closely the sound steel curve than the measured E4 curve. The UT stress is well-predicted for plate E4. Figure 5(a) gives the simulated and measured tensile curves for a second plate with porosity and the sound curve is provided for comparison. Here also the reduction in ductility is well-predicted but not the yield stress. Also, the UT stress is under- 

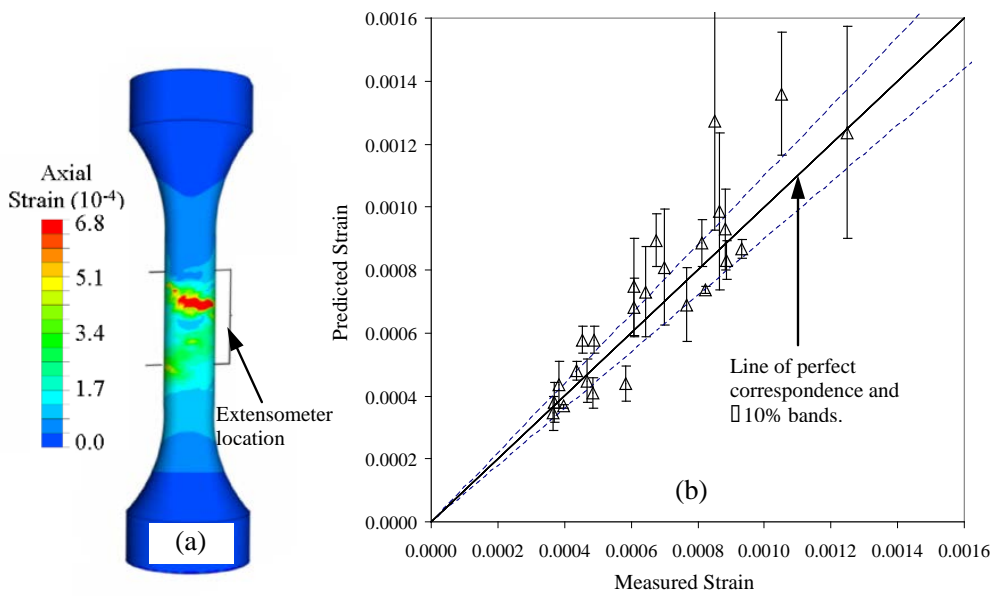

Figure 3 (a) Predicted strain on surface of test specimen, (b) measured and predicted strain from ABAQUS FEA simulations using the $f$ dependent properties for $E$ and $v$.

predicted. In Figure 5(b) a photo of plate after testing compares well with the predicted appearance of the final failure in Figure 5(c). In Figures 6(a) to 6(c) the predicted and measured tensile properties are given. The sound data are circled. In Figure 6(a) the variation in yield stress for the plates with porosity was not predicted, and no prediction was greater than the sound yield. The reason for this is not presently known. The ability to predict ultimate stress and elongation is more encouraging. The elongation appears to have only two outliers where the property is underpredicted. Examining these cases in more detail, especially their porosity mapping results, will lead to refinement of the analysis and improved agreement.

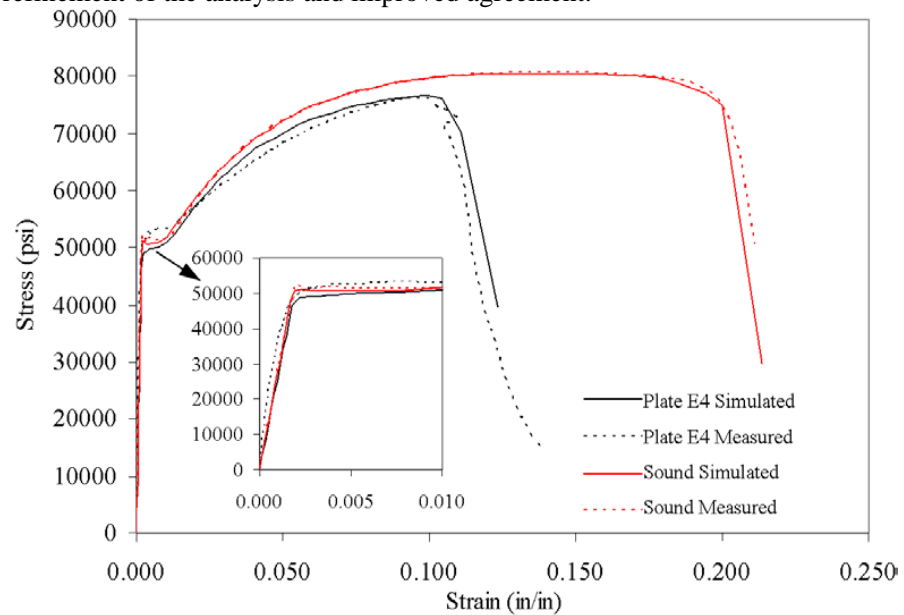

Figure 4 Simulated and measured stress-strain curves for Plate E4 with porosity and sound WCB steel. 

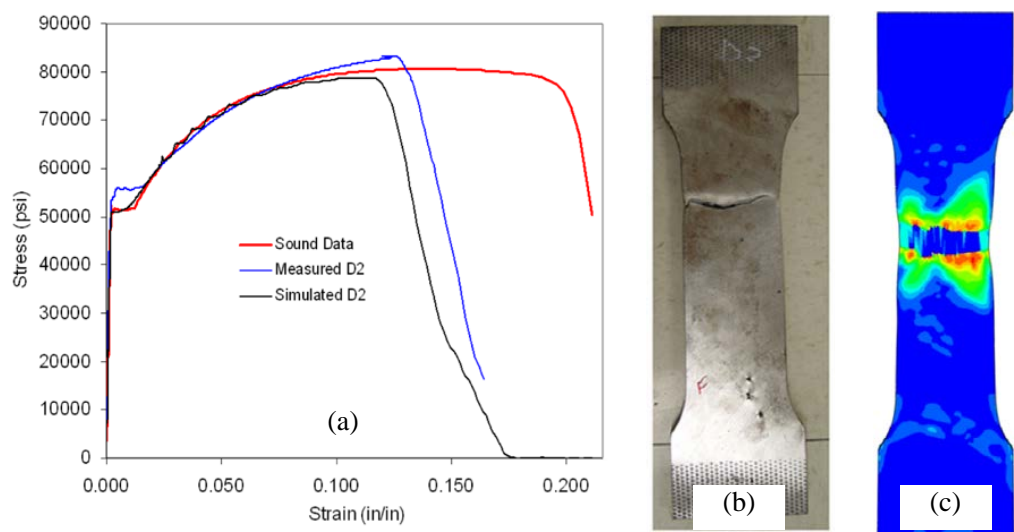

Figure 5 Results for plate-ID D2 (a) Predicted and measured tensile curve, (b) photo of plate after testing, and (c) predicted final failure from Mises stress contour.

\section{Conclusions}

Elastic-plastic behavior of cast steel in the presence of porosity has been predicted using radiographs. The porous metal plasticity material model used here requires nine parameters that were determined for WCB steel. Using these parameters and porosity fields measured from radiographs, the entire tensile test curves for castings with centerline porosity were simulated. Predicted and measured tensile curves and properties were compared. In most cases the comparisons for ultimate stress and elongation are good. Reduction in ductility in the castings is perhaps the most noticeable effect of porosity and it is well-predicted. Disagreement remains between yield stress prediction and measurement. The clearest factors contributing to the disagreement are assumptions and limitations in the model and in determining the porosity distribution in the plate specimen thickness. It is anticipated that this work can be improved by investigating the porosity distribution through the plate thickness further. The castings will be radiographed in their failed condition, to observe the internal damage, comparing the before- and

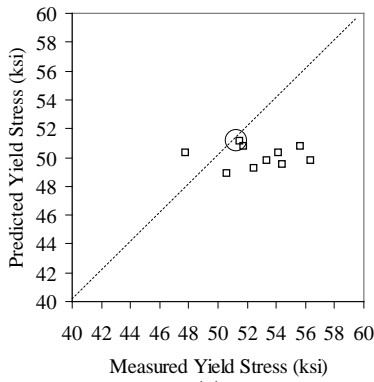

(a)

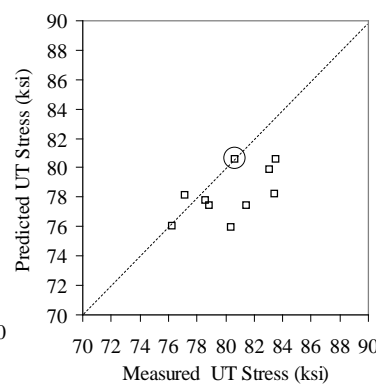

(b)

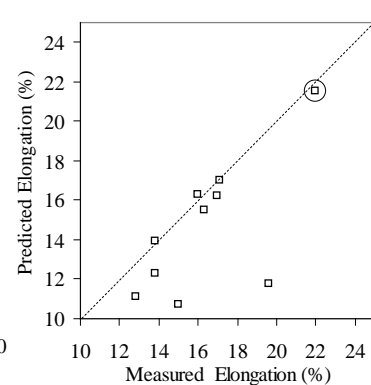

(c)

Figure 6 Predicted and measured tensile properties for sound WCB steel (circled) and for plates with porosity (a) yield stress, (b) ultimate tensile stress, and (c) elongation at fracture. 
after-testing radiographs. Selected sections of the plates will be machined to visualize the through thickness porosity distribution. Using more realistic porosity distributions in the simulations, the accuracy of the model to predict the elastic-plastic response of steel in the presence of porosity can be determined with more certainty.

\section{References}

1. T.L. Anderson, Fracture Mechanics: Fundamentals and Applications, (Boca Raton, FL: Taylor \& Francis, 2005), 219-231.

2. R. Hardin and C. Beckermann, "Effect of Porosity on the Stiffness of Cast Steel," Metall. Mater. Trans. A, 38A (2007), 2992-3006.

3. Hardin, R.A., and Beckermann, C., "Prediction of the Fatigue Life of Cast Steel Containing Shrinkage Porosity," Metall. Mater. Trans. A, 40A (2009), 581-597.

4. P. Heuler, C. Berger, and J. Motz, "Fatigue Behaviour of Steel Castings Containing NearSurface Defects”, Fat. \& Frac. of Eng. Mat. \& Structures, 16 (1992), 115-136.

5. K.M. Sigl, R. Hardin, R.I. Stephens, and C. Beckermann, "Fatigue of 8630 Cast Steel in the Presence of Porosity,” Int. Journal of Cast Metals Research, 17 (2004), 130-146.

6. C.T. Herakovich and S.C. Baxter, "Influence of Pore Geometry on the Effective Response of Porous Media," Journal of Materials Science, 34 (1999), 1595-1609.

7. A.P. Roberts and E.J. Garboczi, "Elastic Properties of Model Porous Ceramics," J. Am. Ceram. Soc., 83 (2000), 3041-3048.

8. E. Zhang and B. Wang, "On the Compressive Behavior of Sintered Porous Coppers with Low to Medium Porosities - Part I: Experimental Study,” Int. J. of Mech. Sci., 47 (2005), 744-756.

9. J.M. Dewey, "The Elastic Constants of Materials Loaded with Non-rigid Fillers," Journal of Applied Physics, 18 (1947), 578-581.

10. A.L. Gurson, "Continuum Theory of Ductile Rupture by Void Nucleation and Growth: Part I - Yield Criteria and Flow Rules for Porous Ductile Materials," Journal of Engineering Materials and Technology, 99 (1977), 2-15.

11. V. Tvergaard, "Influence of Voids on Shear Band Instabilities under Plane Strain Condition," International Journal of Fracture Mechanics, 17 (1981), 389-407.

12. A. Needleman and V. Tvergaard, "An Analysis of Ductile Rupture in Notched Bars," $J$. Mech. Phys. Solids, 32 (1984), 461-490.

13. Dassault Systèmes, ABAQUS Theory Manual, Version 6.10, section 4.3.6 Porous Metal Plasticity (2010).

14. N. Aravas, "On the Numerical Integration of a Class of Pressure-Dependent Plasticity Models,” Int. J. for Num. Methods in Eng., 24 (1987), 1395-1416. 